\title{
Ordered Donor-Acceptor Complex Formation and Electron Transfer in Co-deposited Films of Structurally Dissimilar Molecules
}

Andreas Opitz, a Clea Peter, a Berthold Wegner, ab H.S.S. Ramakrishna Matte, ag Adriana Röttger, a Timo Florian, a Xiaomin Xu, ah Paul Beyer, ai Lutz Grubert, ${ }^{c}$ Stefan Hecht, c Valentina Belova, dk Alexander Hinderhofer, ${ }^{d}$ Frank Schreiber, ${ }^{d}$ Christian Kasper, e Jens Pflaum, e Yadong Zhang, ${ }^{\mathrm{f}}$ Stephen Barlow, ${ }^{\mathrm{f}}$ Seth R. Marder, ${ }^{\mathrm{f}}$ and Norbert Koch ${ }^{\mathrm{ab}}$

a Institut für Physik and IRIS Adlershof, Humboldt-Universität zu Berlin (Germany).

b Helmholtz-Zentrum Berlin für Materialien und Energie (Germany).

c Institut für Chemie and IRIS Adlershof, Humboldt-Universität zu Berlin (Germany).

d Institut für Angewandte Physik, Universität Tübingen (Germany).

e Experimentelle Physik VI, Universität Würzburg (Germany).

$f$ School of Chemistry and Biochemistry and Center for Organic Photonics and Electronics (COPE), Georgia Institute of Technology Atlanta (USA).

g Present address: Energy Materials Laboratory, Centre for Nano and Soft Matter Sciences, Jalahalli, Bengaluru (India)

h Present address: Environmental Science and New Energy Technology Center (Center 1), Shenzhen Geim Graphene Center (SGC), Tsinghua-Berkeley Shenzhen Institute (TBSI), Tsinghua University, (China)

i Present address: Fachbereich Physik, Freie Universität Berlin (Germany)

j Present address: DWI-Leibniz Institute for Interactive Materials, Aachen (Germany) and Institute of Technical and Macromolecular Chemistry, RWTH Aachen University (Germany)

k Present address: Nanostructured Materials Department, Material Science Institute of Barcelona (ICMAB-CSIC, Spain)

Electronic Supplementary Information

\begin{abstract}
Experimental details
Diindenoperylene (DIP) was purchased from Institut für PAH Forschung (Greifenberg, Germany), pentacene (Pen) and dibenzotetrathiafulvalene (DBTTF) from Sigma-Aldrich Chemie $\mathrm{GmbH}$ (Munich, Germany). These materials were used as received. Molybdenum tris(1,2-bis(trifluoromethyl)ethane-1,2-dithiolene) [Mo(tfd $)_{3}$ ] was synthesized and purified as previously described. [1] All films were prepared at room temperature via evaporation of the individual materials in high vacuum (base pressure $<10^{-6} \mathrm{mbar}$ ) from separate resistive heated quartz crucibles. The nominal thickness of each compound was measured separately by quartz-crystal microbalances. The deposition rates were in the range of $1 \mathrm{~nm} \cdot \mathrm{min}^{-1}$. The respective amount of the materials was set due to variation of the individual deposition rates. The ratio used in the manuscript is the molar ratio of the two compounds.
\end{abstract}

Cyclic voltammetry (CV) was performed using a PG310 USB (HEKA Elektronik) potentiostat interfaced to a PC with PotMaster v2x43 (HEKA Elektronik) software for data evaluation. A three-electrode configuration contained in a non-divided cell consisting of a platinum disc $(\mathrm{d}=1 \mathrm{~mm})$ as working electrode, a platinum plate as counter-electrode, and a saturated calomel electrode (SCE) with an agar-agar-plug in a Luggin capillary with a diaphragm as reference electrode was used. Measurements were carried out in $1 \times 10^{-3} \mathrm{M}$ solutions in dichloromethane (DCM, HPLC-grade, dried over calcium hydride and distilled) containing $0.1 \mathrm{M} \mathrm{Bu}_{4} \mathrm{NPF}_{6}$ using a scan rate of $\mathrm{d} U / \mathrm{d} t=1 \mathrm{Vs}^{-1}$. Oxygen was removed by argon purging (Argon $99.999 \%$ ALPHAGAZTM 1 and ALPHAGAZ Purifier, Air Liquide). The data is given in reference to the half-wave potential of the ferrocene redox couple $\left(\mathrm{Fc} / \mathrm{Fc}^{+}\right)$, which was used as external standard. Spectroelectrochemistry was performed in a SEC-C thin layer quartz glass spectroelectrochemical cell $(0.5$ or $1.0 \mathrm{~mm}$ optical path length, working electrode: platinium mesh, counter electrode: platinum wire, reference electrode: non aqueous reference electrode $\mathrm{Ag} / \mathrm{Ag}^{+}$with $0.01 \mathrm{M} \mathrm{AgNO}_{3}$ and $0.1 \mathrm{M}$ $\mathrm{Bu}_{4} \mathrm{NPF}_{6}$ in acetonitrile) from ALS Co. Ltd (Tokyo, Japan) using the Potentiostat PGSTAT 128N (Deutsche Metrohm GmbH \& Co. KG, Filderstadt, Germany) and the software NOVA 2.10. Absorption measurements were performed using the spectrometers AvaSpec-2048x14 with AvaLigth-DH-S-BAL and the software AvaSoft 7.7.2 (both Avantes, Apeldoorn, Netherlands).

Ex situ AFM measurements were performed at a Bruker Dimension Icon in peak force tapping mode (ScanAsyst) using SCANASYST-AIR cantilevers with a typical resonance frequency of $70 \mathrm{kHz}$ and spring constant of $0.4 \mathrm{~N} \cdot \mathrm{m}^{-1}$. Background correction and image analysis were done using the software package Gwyddion. [2] $\vartheta / 2 \vartheta$-scans were performed with an Xray reflectometer $3003 \mathrm{~T} / \mathrm{T}$ by $\mathrm{GE}$ Inspection Technologies using $\mathrm{Cu} \mathrm{K}(\alpha 1)$-radiation $(\lambda=1.54056 \AA)$ with an angular resolution of $0.01^{\circ}$. 
Optical absorption spectroscopy was performed using a Lambda 950 UV/Vis/NIR spectrophotometer (Perkin Elmer Inc.). Measurements in solution were performed in various solvents against filled reference cuvette. Films were prepared on quartz glass (QX-type quartz purchased from Präzisions Glas \& Optik GmbH, Iserlohn, Germany) and measured against an uncoated quartz glass slide.

Raman spectroscopy was performed at different systems based on micro photoluminescence setups and an excitation wavelength of $532 \mathrm{~nm}$ was used in all cases. The films for DBTTF and $\mathrm{Mo}(\mathrm{tfd})_{3}$ as well as the analysed co-deposited films of $\mathrm{DBTTF}$ and $\mathrm{Mo}(\mathrm{tfd})_{3}$ were kept at $77 \mathrm{~K}$ during the measurement in dry nitrogen atmosphere. The other films were measured at room temperature.

Conductivity measurements were carried out for films deposited on a glass substrate with pre-patterned, interdigitated indium tin oxide contacts (channel length $50 \mu \mathrm{m}$, different channel widths due to application of shadow mask) purchased from Ossila Ltd. using a Keithley SourceMeter 2612A in the dark. Several of these in-plane devices (2-5) were measured for each molar ratio.

\section{Absorption spectra of neutral donor molecules in solution}

The results of absorption measurements are shown in Figure S1. DBTTF was dissolved in acetonitrile (MeCN), DIP in dichloromethane (DCM) and pentacene in tetrahydrofuran (THF). Additionally, perylene was measured in MeCN. Absorption maxima and respective molar extinction coefficients are given in Table S1.

\section{Absorption spectra of charged molecules using inorganic dopants in solution}

The absorption features of pristine and charged molecules were studied in solution by charge transfer reactions with inorganic salts. Lithium iodide ( $\mathrm{Lil}, 3 \mathrm{I}^{-} \rightarrow \mathrm{I}_{3}{ }^{-}+2 \mathrm{e}^{-}$) [3] was used to reduce $\mathrm{Mo}(\mathrm{tfd})_{3}$ in chloroform. Pen in toluene and DBTTF in DCM were oxidised by iron(III) chloride $\left(\mathrm{FeCl}_{3}, \mathrm{Fe}^{3+}+\mathrm{e}^{-} \rightarrow \mathrm{Fe}^{2+}\right)$.[4] The results are visualised in Figure S2. Absorption features of the $\mathrm{Mo}(\mathrm{tfd})_{3}$ anion [5,6], the Pen cation [6,7], and the DBTTF cation [8] were clearly distinguishable from the absorption features of the neutral molecules in all three cases as reported in literature before and as shown by spectroelectrochemistry data below. The absorption of Pen is constant upon adding the oxidising agent in the saturated Pen solution, because additional neutral molecules were dissolved upon oxidising the dissolved Pen molecules. A reduction of absorption intensity of the neutral species and an increase of absorption intensity for the charged species are visible for $\mathrm{Mo}(\mathrm{tfd})_{3}$ and DBTTF upon adding the inorganic salt, for the Pen cation only the raise of absorption is observed.

No absorption of cationic DIP molecules were observed upon addition of ceric ammonium nitrate (CAN, $\mathrm{Ce}^{4+}+\mathrm{e}^{-} \rightarrow \mathrm{Ce}^{3+}$ ) $[9,10]$ solution or $\mathrm{FeCl}_{3}$ solution (not shown) to a DIP solution in acetone. CAN was used as oxidising agent showing a reduction potential, which is by $0.76 \mathrm{~V}$ higher than that of $\mathrm{FeCl}_{3}$. [10] The observed decrease of absorption intensity can be described by dilution due to the added solvent for $\mathrm{FeCl}_{3}$ and is stronger than dilution for CAN.

\section{Cyclic voltammetry and spectroelectrochemistry of donor molecules in solution}

For the donor molecules discussed here, cyclic voltammetry (CV) and spectroelectrochemistry (SEC) were performed in solution. As DIP and Pen are poorly soluble in organic solvents, more effort was necessary to study these materials. Perylene was used in comparison to DIP and Pen was characterised by differential pulsed voltammetry (DPV).

DBTTF: Figure S3 shows the CV measured curves for the oxidation of DBTTF dissolved in DCM and MeCN. The oxidation potential was determined to be $0.11 \mathrm{~V}$ as a standard potential. The absorption spectra upon charging of DBTTF are given in Figure S4. The left part indicates the formation of the radical cation, the right part the formation of the di-cation. The changes of spectral absorption are specified as 3D plot in Figure S5. Figure S6 summarizes the absorption spectra of neutral DBTTF, the radical cation $\mathrm{DBTTF}^{+}$and the di-cation $\mathrm{DBTTF}^{++}$.

DIP: Figure S7 shows the CV measured curves for the oxidation and reduction of DIP dissolved in DCM. The determined standard potential for the oxidation of $0.41 \mathrm{~V}$ is discussed later with respect to the changes of the absorption spectra. The absorption spectra during oxidation and reduction of DIP are given in Figure S8. The intensity of DIP absorption features are decreasing and additional absorption features below the absorption edge of the neutral molecule, as observed during oxidation of DBTTF and reduction of DIP, are absent. This is the same observation as for DIP upon adding inorganic salts. The changes of spectral absorption and difference spectra for DIP oxidation are specified as 3D plot in Figure S9. As the absorption spectra before and after the cycling are comparable, the process seems to be reversible even without absorption features of the DIP radical cation. In comparison to the poorly soluble DIP, the oxidation potential for the dibenzotetraphenylperiflanthene was determined as $E_{1 / 2}=0.51 \mathrm{~V}$ vs. $\mathrm{FeCp}_{2} / \mathrm{FeCp}_{2}{ }^{+}\left(0.1 \mathrm{M} \mathrm{Bu}_{4} \mathrm{NPF}_{6}\right.$ in MeCN:toluene $\left.=1: 1\right)$. [11]

Perylene: Due to the absence of absorption features of the DIP radical cation during SEC measurements, perylene was measured also. Figure S10 shows the CV measured curves for the oxidation and reduction of perylene dissolved in DCM. Here only peak potentials are given. The absorption spectra during oxidation and reduction of perylene are given in Figure S11. The changes of spectral absorption for perylene reduction and oxidation are specified as 3D plot in Figure S12. The data are comparable to values in the literature. [12] 
Pentacene: Table S2 contains potentials for pentacene found in literature. The data were determined by different methods (CV, DPV). Mainly ortho-dichlorobenzene was used as a solvent. Due to the variation of literature values pentacene was measured under similar condition as the other donors here. DPV was performed instead of CV because of low solubility of pentacene as shown in Figure S13. The oxidation potential was determined to be $0.21 \mathrm{~V}$ as a standard potential. Additionally the DPV curves is shown for a heated solution $\left(\approx 60^{\circ} \mathrm{C}\right)$ for comparison.

\section{Discussion on the oxidation of perylene and DIP}

Reversible oxidation was observed for perylene, reversible reduction took place for perylene and DIP. The formation of perylene anions and cations as well as DIP anions was confirmed by absorption spectra upon electrochemical charging, as shown in Figure S3. [12-15]

An increase of the current appears in the voltage range, where the oxidation of DIP is expected. However, the absorption spectra show a decrease of absorption of the neutral molecules and absorption features of the DIP cations or other reaction products are absent as observed also for adding the inorganic salt CAN. This behaviour hints on an additional reaction present upon oxidation and condensation of the insoluble product onto the electrode. The resulting potential onset is $+0.47 \mathrm{~V}$ (vs. $\left.\mathrm{Fc} / \mathrm{Fc}^{+}\right)$and the half-wave potential $+0.41 \mathrm{~V}\left(\mathrm{vs}\right.$. $\left.\mathrm{Fc} / \mathrm{Fc}^{+}\right)$in dichloromethane. The rapid follow-up reaction results in a shifted standard potential by up to $350 \mathrm{meV}$ as estimated by the Nernst equation for the complete absence of absorption features of DIP cations. The observation of DIP anions and the impossibility to generate DIP cations was described before for molecules in solution without giving further details. [15] However, this electrochemical reaction is reversible. The condensed product will react back to DIP upon electron addition. The absorption spectrum of DIP is recovered after cycling the voltage ramp as shown with Figure S9. We propose a dimerization with subsequent deposition on the platinum mesh electrode as observed also for other polycyclic hydrocarbons. [16]

\section{Absorption spectra of DIP films with inorganic salts}

The electron transfer between DIP and $\mathrm{FeCl}_{3}$ as well as between DIP and CAN was analysed in thin films. The films were produced either by co-deposition in vacuum ( $\mathrm{vac}, \mathrm{DIP}+\mathrm{FeCl}_{3}$ ) or by sequential deposition (sqd) of the dissolved salt on an separately deposited DIP film (DIP+FeCl 3 and DIP+CAN). The absorption spectra are shown in Figure S14. Due to adding the inorganic salt, new absorption features below the absorption edge of the DIP film are detected. The features at about 0.94 and $1.48 \mathrm{eV}$ were reported before. [17] An additional feature is detected here at an energy below $0.60 \mathrm{eV}$. As all three feature are obtainable upon adding different oxidising agents and deposition methods, we propose the presence of DIP cations in these films. The follow-up reaction observed in solution is prevented presumably due to close packing of the molecules and therefore steric hindered in the solid film.

\section{Energy levels determined by direct and inverse photoelectron spectroscopy}

Ionization energy and electron affinity measured by direct and inverse photoelectron spectroscopy on thin films were reported in literature and are summarized in Figure S15. [5,18-20] IEs for DIP and Pen are given for molecules oriented in more lying or standing fashion with respect to the substrate; the value for DBTTF is reported only for standing orientation. Analysis of orientation dependent EA values for $\mathrm{Mo}(\mathrm{tfd})_{3}$ is missing. The IE of DIP with lying molecules is higher than the EA of $\mathrm{Mo}(\mathrm{tfd})_{3}$, which would forbid electron transfer from DIP to $\mathrm{Mo}(\mathrm{tfd})_{3}$. This gives a similar scenario as with the values determined by CV measurements. All other donor IEs are lower than the EA of $\mathrm{Mo}(\mathrm{tfd})_{3}$ and electron transfer would be possible.

\section{Supplementary structural data: $x$-ray reflectivity data and scanning force micrographs}

X-ray reflectivity data for a separately deposited DIP film and an equimolar co-deposited DIP:Mo(tfd) ${ }_{3}$ one are given in Figure S16. The (001) reflection resulting from crystalline DIP in the $\sigma$-phase is clear observed in both spectra. [21] The absence of the (002) peak and broadening of the (001) peak together with the loss of Laue fringes measured for the codeposited film show a reduced coherence length perpendicular to the substrate surface. However, the crystalline phase of DIP is persisting upon adding with $\mathrm{Mo}(\mathrm{tfd})_{3}$.

The scanning force micrograph for a co-deposited film of DBTTF and $\mathrm{Mo}(\mathrm{tfd})_{3}$ with a molar ratio of 4:1 is shown in Figure S17. The images show the two different morphologies of DBTTF:Mo(tfd $)_{3}$ films as discussed in the main text. The high crystallites were observed in the equimolar co-deposited films and the large area and related to the ordered complex, flat crystallites were described for the co-deposited film with a molar ratio of 10:1. The presence of these two different morphologies supports the description of two intendent morphologies and shows the transition between these morphologies.

\section{Absorption of DBTTF and $\mathrm{Mo}(\mathrm{tfd})_{3}$ in solution}

Absorption spectra were taken for DBTTF solution upon adding $\mathrm{Mo}(\mathrm{tfd})_{3}$ solution and for $\mathrm{Mo}(\mathrm{tfd})_{3}$ solution. The results are shown in Figure S18. The used solvent is DCM. By comparing with the spectra measured upon doping with inorganic salts (see Figure S2), the appearing features are related to the formation of $\mathrm{Mo}(\mathrm{tfd})_{3}$ anions and DBTTF cations. Nearly complete ionisation of added $\mathrm{Mo}(\mathrm{tfd})_{3}$ seems to be present for molar ratios of 20:1 and 10:1. For higher acceptor contents also the absorption features of neutral $\mathrm{Mo}(\mathrm{tfd})_{3}$ become visible. 
Supporting figures

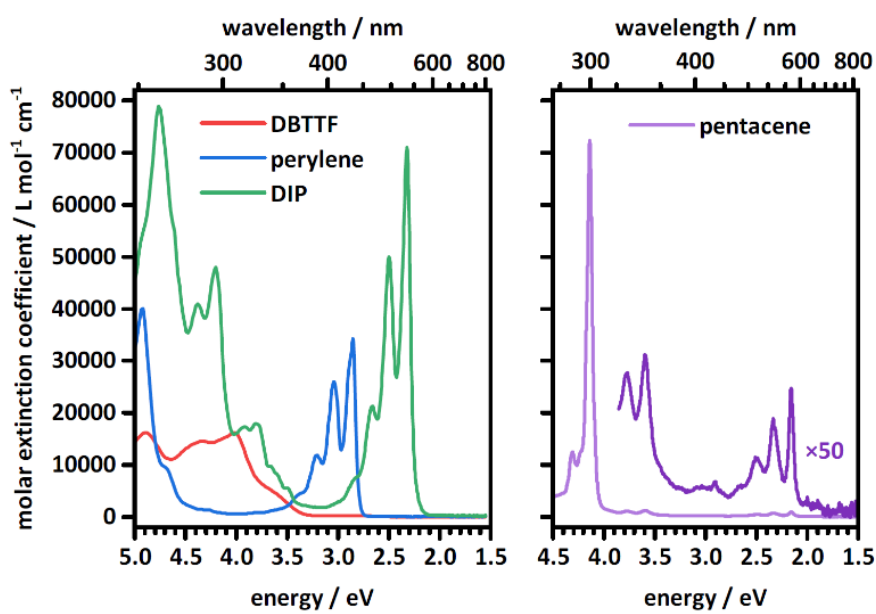

Figure S1: UV-vis-NIR absorption spectra of pristine compounds used in solution.

Table S1: Wavelength of absorption maxima and respective molar extinction coefficient.

\begin{tabular}{|c|c|c|c|c|c|c|c|}
\hline \multirow{2}{*}{$\begin{array}{c}\text { material } \\
\text { DBTTF }\end{array}$} & \multirow{2}{*}{$\begin{array}{c}\text { solvent } \\
\mathrm{MeCN}\end{array}$} & \multicolumn{6}{|c|}{$\lambda$ in $\mathrm{nm} \mid \varepsilon$ in $\mathrm{L} \mathrm{mol}^{-1} \mathrm{~cm}^{-1}$} \\
\hline & & 253 & 16000 & 286 & 14500 & 308 & 16.000 \\
\hline perylene & $\mathrm{MeCN}$ & 386 & 12000 & 407 & 26000 & 434 & 34.500 \\
\hline DIP & DCM & 465 & 21000 & 496 & 50000 & 534 & 71.000 \\
\hline pentacene & THF & 300 & 73000 & 345 & 1255 & 574 & 990 \\
\hline
\end{tabular}



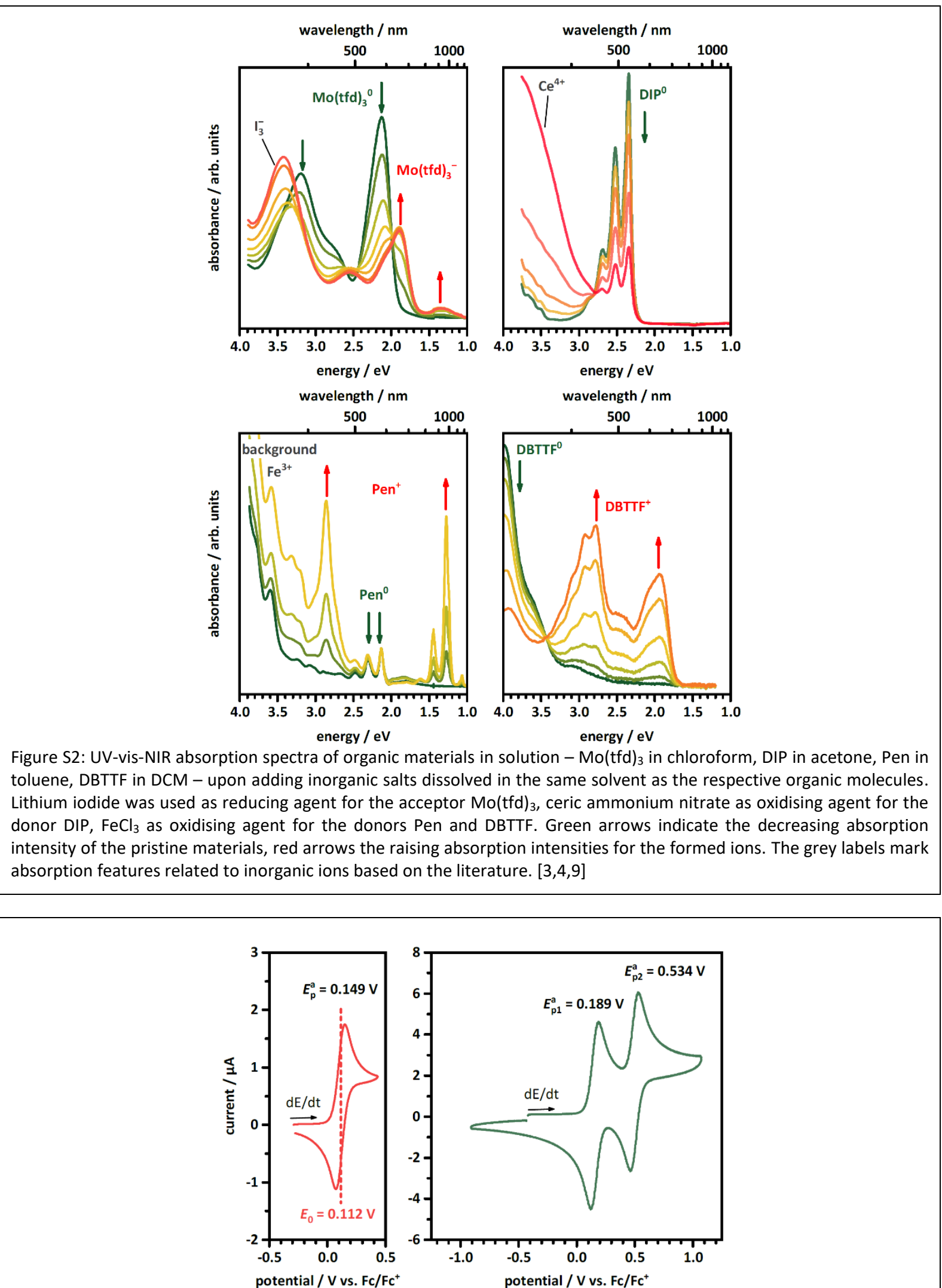

Figure S3: CV current-voltage curves of DBTTF ( $c=1 \cdot 10^{-3} \mathrm{~mol} / \mathrm{l}$, Pt-disc $d=1 \mathrm{~mm}$ ) dissolved (left) in DCM with $0.1 \mathrm{M} \mathrm{Bu}_{4} \mathrm{NPF}_{6}$ as electrolyte, scan rate $\mathrm{d} U / \mathrm{d} t=100 \mathrm{mV} / \mathrm{s}$ and (right) in $\mathrm{MeCN}$ with $0.1 \mathrm{M} \mathrm{Bu}_{4} \mathrm{NPF}_{6}$, scan rate $\mathrm{d} U / \mathrm{d} t=1 \mathrm{~V} / \mathrm{min}$. 

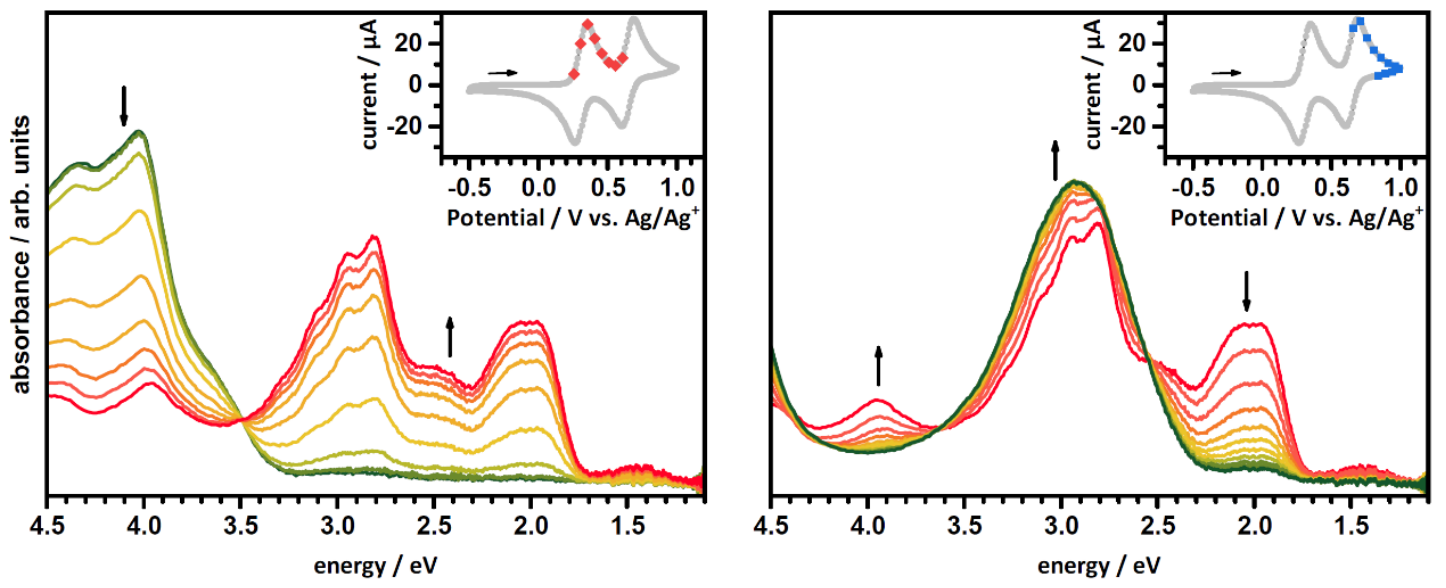

Figure S4: Changes in the UV-vis absorption spectra of DBTTF ( $c=5 \cdot 10^{-4} \mathrm{~mol} / \mathrm{I}$ in DCM with $0.1 \mathrm{M} \mathrm{Bu}_{4} \mathrm{NPF}_{6}$ ). Left: build-up of the radical cation $\mathrm{DBTTF}^{++}$in the voltage range of $0.25 \rightarrow 0.61 \mathrm{~V}\left[\mathrm{vs}\right.$. Ag/AgNO 3 . Right: build-up of the di-cation DBTTF ${ }^{++}$ over the voltage range $0.66 \rightarrow 1.00 \rightarrow 0.84 \mathrm{~V}$ [vs. $\mathrm{Ag} / \mathrm{AgNO}_{3}$ ]. Parameter for both: scan rate $\mathrm{dU} / \mathrm{d} t=10 \mathrm{mV} / \mathrm{s}$, quartz cuvette $d=0.5 \mathrm{~mm}$. The colour points in the insets show at which current-voltage pairs absorption spectra were recorded.
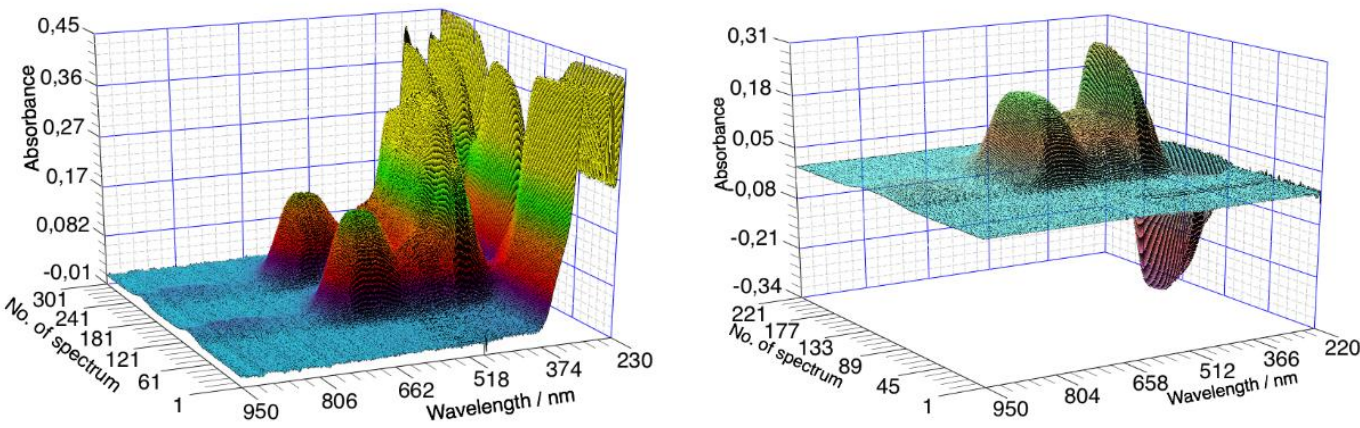

Figure S5: Changes in the UV-vis spectra of DBTTF during cyclic voltammetry shown in Figure S4 as 3D plots. Left: absorption spectra in the voltage range of $-0.50 \rightarrow 1.00 \rightarrow-0.50 \mathrm{~V} \rightarrow 0.0 \mathrm{~V}$ [vs. $\mathrm{Ag} / \mathrm{AgNO}_{3}$ ], observed species DBTTF $\rightarrow$

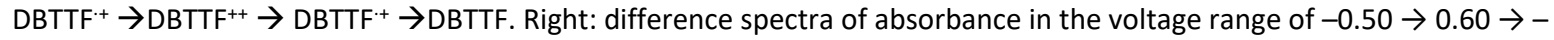
$0.50 \mathrm{~V}\left[\mathrm{vs} . \mathrm{Ag} / \mathrm{AgNO}_{3}\right]$, observed species DBTTF $\rightarrow \mathrm{DBTTF}^{+} \rightarrow$ DBTTF.

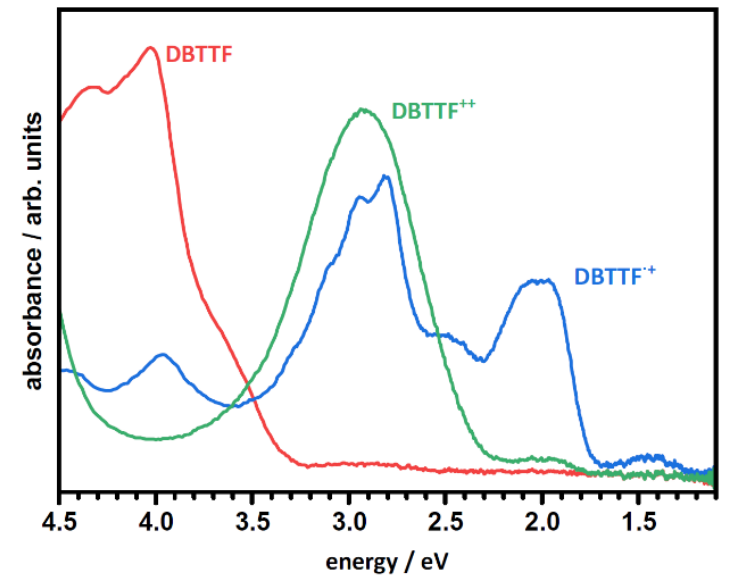

Figure S6: UV-vis absorption spectra of compounds DBTTF, DBTTF++ and DBTTF ${ }^{++}$taken from Figure S4. 


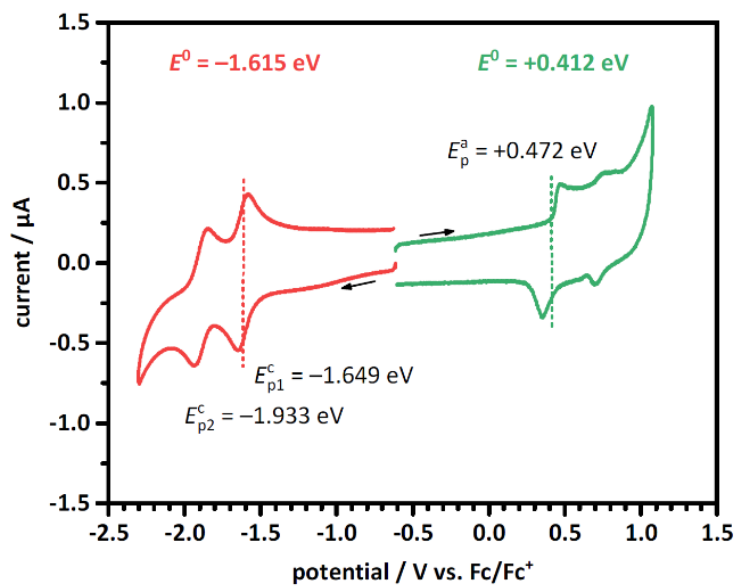

Figure S7: CV current-voltage curves of DIP $\left(c=1 \cdot 10^{-4} \mathrm{~mol} / \mathrm{l}, \mathrm{Pt}\right.$-disc $d=1 \mathrm{~mm}$ ) dissolved in DCM with $0.1 \mathrm{M} \mathrm{Bu}_{4} \mathrm{NPF}_{6}$ as electrolyte, scan rate $\mathrm{d} U / \mathrm{d} t=100 \mathrm{mV} / \mathrm{s}$.
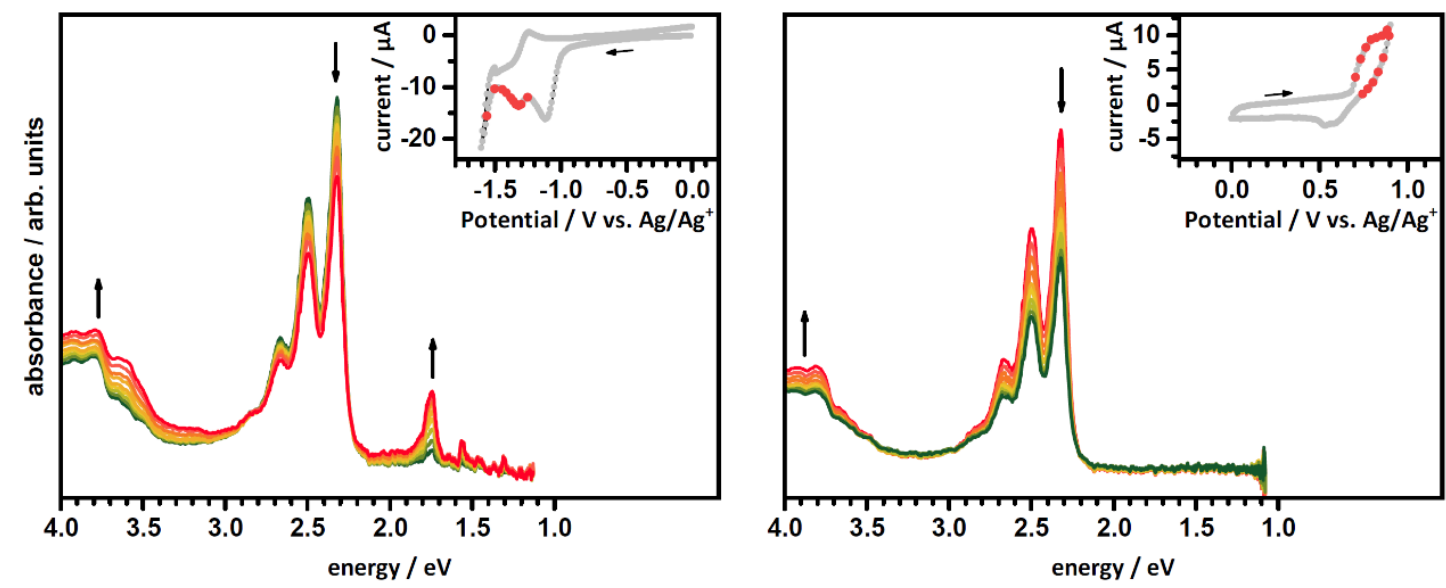

Figure S8: Changes in the UV-vis absorption spectra of DIP ( $\mathrm{c}=5 \cdot 10^{-5} \mathrm{~mol} / \mathrm{I}$ in DCM with $0.1 \mathrm{M} \mathrm{Bu}_{4} \mathrm{NPF}_{6}$ ) for the reduction (left) and oxidation (right). Parameters: scan rate $\mathrm{d} U / \mathrm{d} t=10 \mathrm{mV} / \mathrm{s}$, quartz cuvette $d=1 \mathrm{~mm}$. The colour points in the insets show at which current-voltage pairs absorption spectra were recorded.
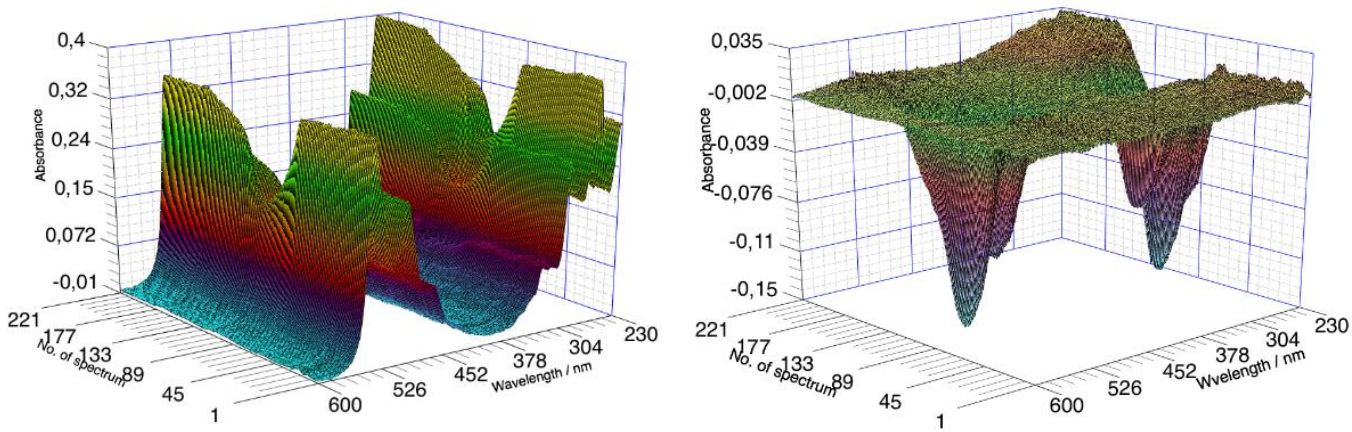

Figure S9: Changes in the UV-vis spectra of DIP during cyclic voltammetry shown in Figure S8 (right part) as 3D-Plot. Left: absorption spectra in the voltage range of $0.0 \rightarrow 0.90 \rightarrow-0.20 \mathrm{~V} \rightarrow 0.0 \mathrm{~V}$ [vs. $\left.\mathrm{Ag} / \mathrm{AgNO}_{3}\right]$. Right: difference spectra of absorbance in the same voltage range. 


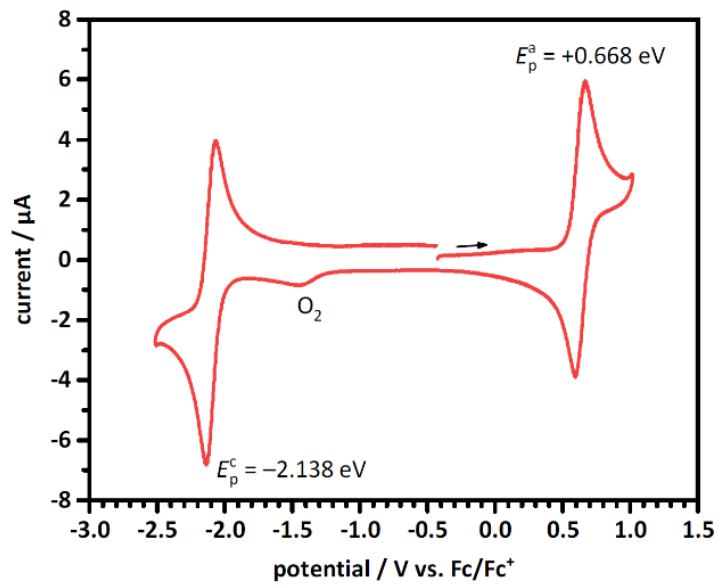

Figure S10: CV current-voltage curves of perylene $\left(c=1 \cdot 10^{-3} \mathrm{~mol} / \mathrm{l}\right.$, Pt-disc $\left.d=1 \mathrm{~mm}\right)$ dissolved in MeCN with $0.1 \mathrm{M} \mathrm{Bu}_{4} \mathrm{NPF}_{6}$ as electrolyte, scan rate $\mathrm{d} U / \mathrm{d} t=1 \mathrm{~V} / \mathrm{s}$.
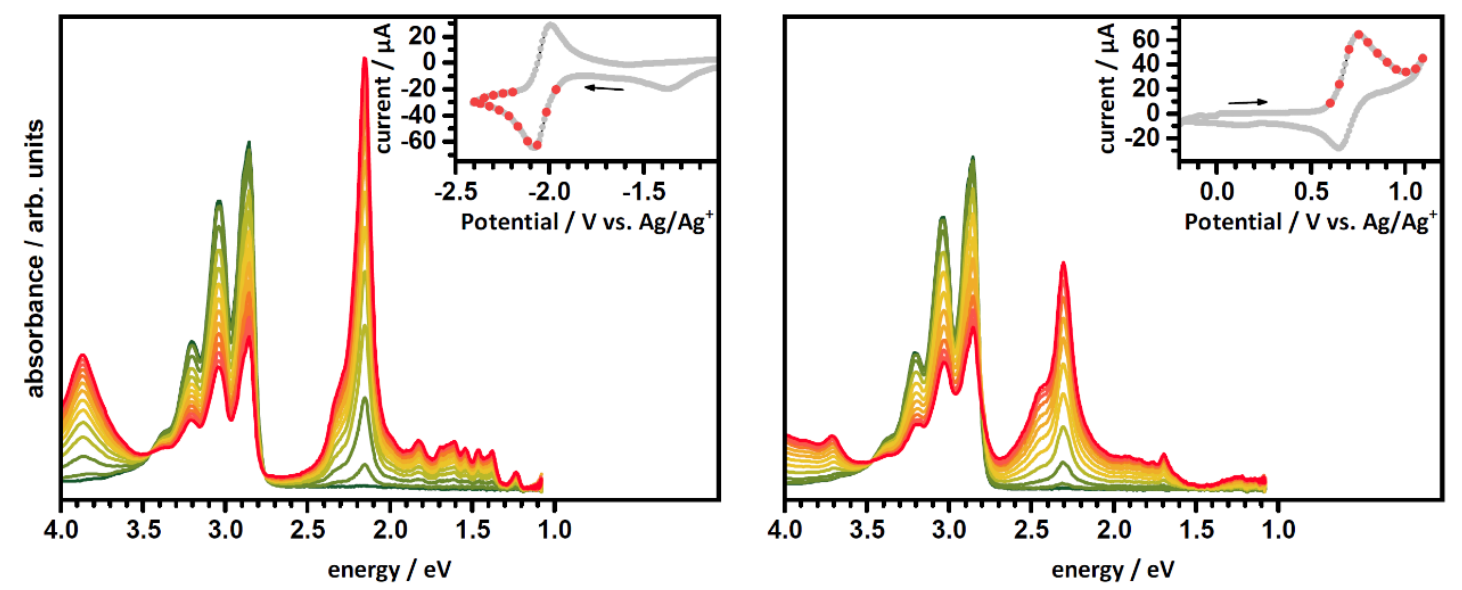

Figure S11: Changes in the UV-vis absorption spectra of perylene ( $c=3.86 \cdot 10^{-4} \mathrm{~mol} / \mathrm{l}$ in MeCN with $\left.0.1 \mathrm{M} \mathrm{Bu}_{4} \mathrm{NPF}_{6}\right)$ for the reduction (left) and oxidation (right). Parameters: scan rate $\mathrm{d} U / \mathrm{d} t=10 \mathrm{mV} / \mathrm{s}$, quartz cuvette $d=1 \mathrm{~mm}$. The colour points in the insets show at which current-voltage pairs absorption spectra were recorded. 

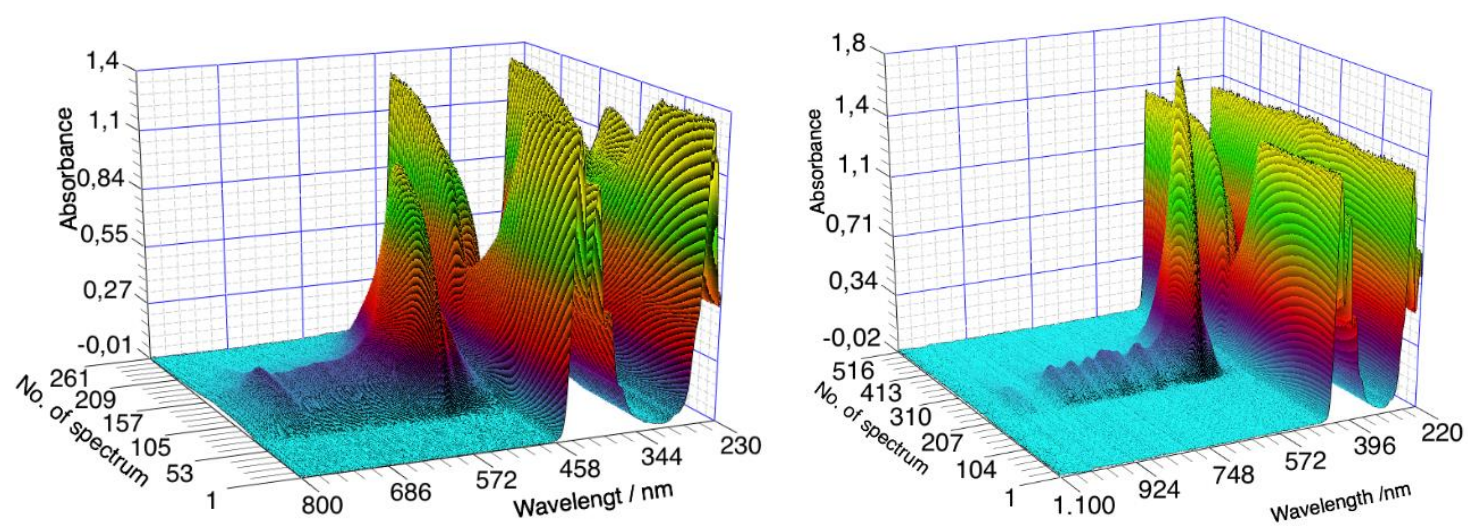

Figure S12: Changes in the UV-Vis spectra of perylene during cyclic voltammetry shown in Figure S11 for reduction (left, voltage range $0 \mathrm{~V} \rightarrow-2.40 \mathrm{~V} \rightarrow 0.20 \rightarrow 0 \mathrm{~V}\left[\mathrm{vs} . \mathrm{Ag} / \mathrm{AgNO}_{3}\right]$ ) and oxidation (right, voltage range $0 \mathrm{~V} \rightarrow 1.10 \mathrm{~V} \rightarrow-0.20 \rightarrow$ $\left.0 \mathrm{~V}\left[\mathrm{vs} . \mathrm{Ag} / \mathrm{AgNO}_{3}\right]\right)$.

Table S2: Literature survey for pentacene oxidation potentials.

\begin{tabular}{|c|c|c|c|c|c|c|}
\hline & potential / V & $\begin{array}{l}\text { reference } \\
\text { electrode }\end{array}$ & solvent $^{1}$ & comment & method & reference \\
\hline & 0.10 & $\mathrm{Fc} / \mathrm{Fc}^{+}$ & DCM & $E_{\mathrm{p}}^{\mathrm{a}}$ & $\mathrm{CV}$ & [22] \\
\hline & 0.27 & Pseudo-Ag & oDCB & $E_{\text {onset }} @ 100{ }^{\circ} \mathrm{C}$ & $\mathrm{CV}$ & [23] \\
\hline & 0.30 & $\mathrm{Fc} / \mathrm{Fc}^{+}$ & oDCB & $E^{0}$ & DPV, CV & [24] \\
\hline & 0.22 & $\mathrm{Fc} / \mathrm{Fc}^{+}$ & oDCB & $E_{\mathrm{p}}^{\mathrm{a}}$ & DPV & [25] \\
\hline & $0.64(0.24 *)$ & $\mathrm{SCE}\left(\mathrm{Fc} / \mathrm{Fc}^{+}\right)$ & oDCB & $E_{\text {onset }}$ & $\mathrm{CV}$ & [26] \\
\hline & 0.28 & $\mathrm{Fc} / \mathrm{Fc}^{+}$ & DCM & $E^{0}$ & $\mathrm{CV}$ & [27] \\
\hline & $0.70\left(0.30^{*}\right)$ & $\mathrm{SCE}\left(\mathrm{Fc} / \mathrm{Fc}^{+}\right)$ & ODCB & $E_{1 / 2}$ & $\mathrm{CV}$ & [28] \\
\hline & $.82 * *(0.42 *)$ & $\mathrm{SCE}\left(\mathrm{Fc} / \mathrm{Fc}^{+}\right)$ & & & & [29] \\
\hline & 0.22 & $\mathrm{Fc} / \mathrm{Fc}^{+}$ & oDCB & $E^{0}$ & DPV & [30] \\
\hline & 0.37 & $\mathrm{Fc} / \mathrm{Fc}^{+}$ & ODCB & $E^{0}$ & average & [31] \\
\hline \multicolumn{7}{|c|}{$\begin{array}{l}\text { conversion to reference electrode } \mathrm{Fc} / \mathrm{Fc}^{+} \text {; } \\
\text { not measured: „The value for pentacene has been derived from linear plot of oxidation } \\
\text { potential against the coefficient of the highest occupied molecular orbital in the series of } \\
\text { linear polyacenes benzene to tetracene extended to pentacene." }\end{array}$} \\
\hline \multicolumn{7}{|c|}{ DCM - dichloromethane, oDCB - ortho-dichlorobenzene } \\
\hline 2 & \multicolumn{6}{|c|}{$\begin{array}{l}\text { CV - cyclic voltammetry, DPV - differential pulsed voltammetry, average - average of } \\
\text { literature values }\end{array}$} \\
\hline
\end{tabular}




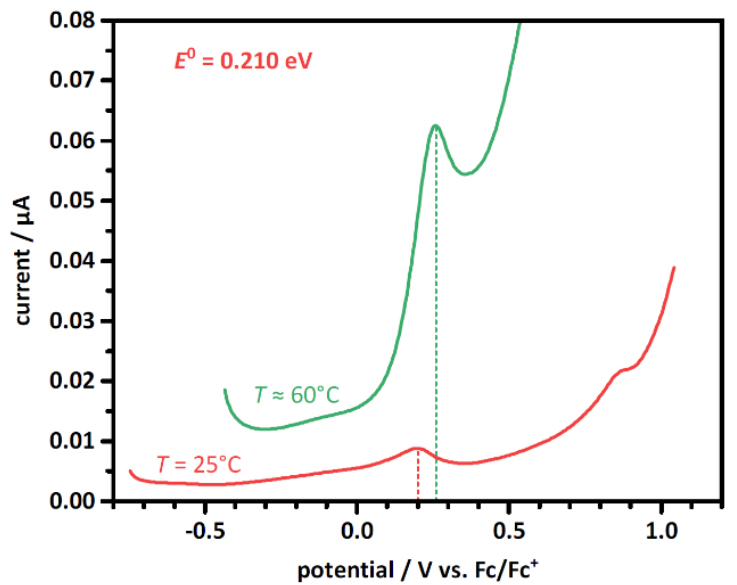

Figure S13: DPV current-voltage curves for pentacene saturated in ortho-DCB with $0.075 \mathrm{M} \mathrm{Bu}_{4} \mathrm{NPF}_{6}$. Parameters: Pt-disc $d=1 \mathrm{~mm}$, modulation amplitude $25 \mathrm{mV}$.

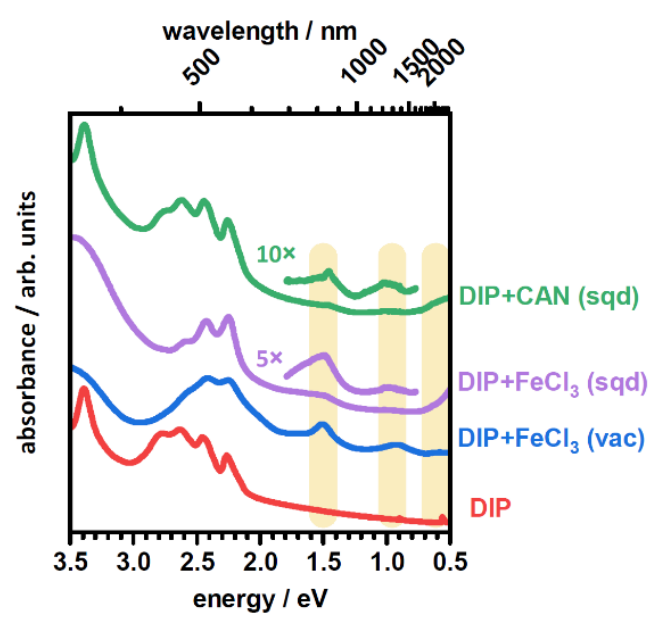

Figure S14: Absorption spectra of DIP films [separately deposited, co-deposited with $\mathrm{FeCl}_{3}(\mathrm{vac})$ and sequentially deposited $\mathrm{FeCl}_{3}$ and $\mathrm{CAN}$ (sqd)]. New absorption features appear below $0.60 \mathrm{eV}$ as well as about 0.94 and $1.48 \mathrm{eV}$, which are absent for the DIP film. The zoomed curved are shown after parabolic background subtraction for better visibility of the peaks. 


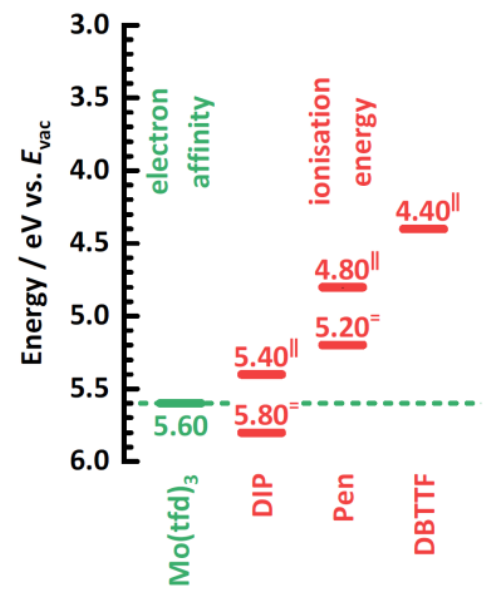

Figure S15: Ionisation energies and electron affinity taken from literature. [5,18-20] IEs for DIP and Pen are given for molecules oriented in more lying (=) or standing (II) fashion with respect to the substrate.

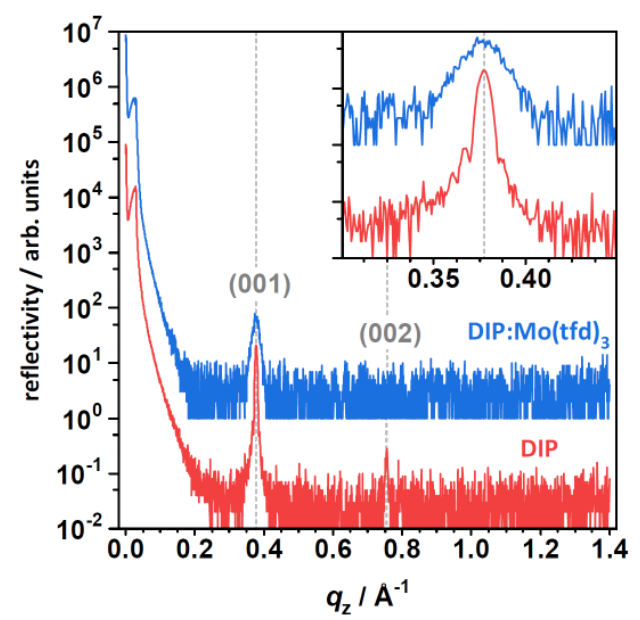

Figure S16: X-ray reflectivity data for pristine DIP and equimolar co-deposited DIP:Mo(tfd) ${ }_{3}$ film. The grey dashed lines give the positions of (001) and (002) reflections for thin DIP films in the $\sigma$-phase. The inset in the upper right corner shows a zoom of the (001) feature.

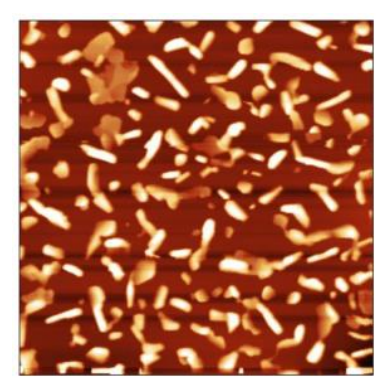

Figure S17: Scanning force micrographs for a co-deposited films of DBTTF and Mo(tfd $)_{3}$ with a molar ratio of about 4:1. The size for all images is $4 \times 4 \mu \mathrm{m}^{2}$. 


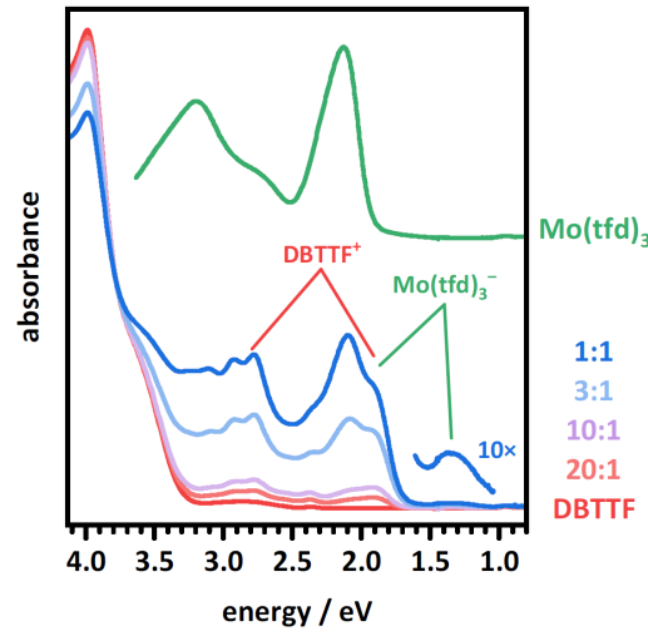

Figure S18: Absorption measured during titration of DBTTF solution by adding $\mathrm{Mo}(\mathrm{tfd})_{3}$ solution. The molar equivalents are given. Additionally, the absorption of $\mathrm{Mo}(\mathrm{tfd})_{3}$ in solution is shown.

\section{References}

[1] A. Davison, R.H. Holm, R.E. Benson, W. Mahler, Metal Complexes Derived from cis -1,2-dicyano-1,2ethylenedithiolate and Bis(Trifluoromethyl)-1,2-dithiete, in: Inorg. Synth. Vol. 10, John Wiley \& Sons, Ltd, 2007: pp. 8-26. doi:10.1002/9780470132418.ch3.

[2] D. Nečas, P. Klapetek, Gwyddion: an open-source software for SPM data analysis, Open Phys. 10 (2012) 181-188. doi:10.2478/s11534-011-0096-2.

[3] S. V Kireev, S.L. Shnyrev, Study of molecular iodine, iodate ions, iodide ions, and triiodide ions solutions absorption in the UV and visible light spectral bands, Laser Phys. 25 (2015) 075602. doi:10.1088/1054-660X/25/7/075602.

[4] I. Fontana, A. Lauria, G. Spinolo, Optical absorption spectra of Fe2+ and Fe3+ in aqueous solutions and hydrated crystals, Phys. Status Solidi B. 244 (2007) 4669-4677. doi:10.1002/pssb.200743103.

[5] Y. Qi, T. Sajoto, S. Barlow, E.-G. Kim, J.-L. Brédas, S.R. Marder, A. Kahn, Use of a High Electron-Affinity Molybdenum Dithiolene Complex to p-Dope Hole-Transport Layers, J. Am. Chem. Soc. 131 (2009) 12530-12531. doi:10.1021/ja904939g.

[6] B. Wegner, Molecular doping of organic semiconductors - contributions to its basic understanding and application, doctoral thesis at Humboldt-Universität zu Berlin, 2019.

[7] J. Szczepanski, C. Wehlburg, M. Vala, Vibrational and electronic spectra of matrix-isolated pentacene cations and anions, Chem. Phys. Lett. 232 (1995) 221-228. doi:10.1016/0009-2614(94)01340-2.

[8] P. Beyer, D. Pham, C. Peter, N. Koch, E. Meister, W. Brütting, L. Grubert, S. Hecht, D. Nabok, C. Cocchi, C. Draxl, A. Opitz, State-of-Matter-Dependent Charge-Transfer Interactions between Planar Molecules for Doping Applications, Chem. Mater. 31 (2019) 1237-1249. doi:10.1021/acs.chemmater.8b01447.

[9] G. Özen, B. Demirata, Energy transfer characteristics of the hydrogen peroxide induced Ce3+-Ce4+ mixture, Spectrochim. Acta Part A Mol. Biomol. Spectrosc. 56 (2000) 1795-1800. doi:10.1016/S1386-1425(00)00237-7.

[10] G.R. Bamwenda, T. Uesigi, Y. Abe, K. Sayama, H. Arakawa, The photocatalytic oxidation of water to O2 over pure CeO2, WO3, and TiO2 using Fe3+ and Ce4+ as electron acceptors, Appl. Catal. A Gen. 205 (2001) 117-128. doi:10.1016/S0926-860X(00)00549-4.

[11] J.D. Debad, J.C. Morris, V. Lynch, P. Magnus, A.J. Bard, Dibenzotetraphenylperiflanthene: Synthesis, Photophysical Properties, and Electrogenerated Chemiluminescence, J. Am. Chem. Soc. 118 (1996) 2374-2379. doi:10.1021/ja9537888.

[12] M. Svaan, V.D. Parker, V.D. Parker, P. Krogsgaard-Larsen, U. Örn, Temperature Effects on Electrode Processes. I. The Entropy of the Reversible Oxidation and Reduction of Alternant Aromatic Hydrocarbons., Acta Chem. Scand. 35b (1981) 559-565. doi:10.3891/acta.chem.scand.35b-0559.

[13] T. Aruga, O. Ito, M. Matsuda, Photoinduced electron transfer from anions. Part 2. Formation and decay of radical anions of aromatic compounds produced by photoinduced electron transfer from the triphenylstannyl anion, J. Phys. Chem. 86 (1982) 2950-2953. doi:10.1021/j100212a028.

[14] J. Guo, T. Togami, H. Benten, H. Ohkita, S. Ito, Simultaneous multi-photon ionization of aromatic molecules in polymer solids with ultrashort pulsed lasers, Chem. Phys. Lett. 475 (2009) 240-244. doi:10.1016/j.cplett.2009.05.070.

[15] Y. Kubozono, M. Ata, Y. Gondo, Electronic structure of diindeno[1,2,3-cd:1',2',3'-Im]perylene radical anion, Spectrochim. Acta Part A Mol. Spectrosc. 46 (1990) 57-59. doi:10.1016/0584-8539(93)80011-X.

[16] T. Beitz, R. Laudien, H.-G. Löhmannsröben, B. Kallies, Ion Mobility Spectrometric Investigation of Aromatic Cations 
in the Gas Phase, J. Phys. Chem. A. 110 (2006) 3514-3520. doi:10.1021/jp055335n.

[17] G. Duva, L. Pithan, C. Zeiser, B. Reisz, J. Dieterle, B. Hofferberth, P. Beyer, L. Bogula, A. Opitz, S. Kowarik, A. Hinderhofer, A. Gerlach, F. Schreiber, Thin-Film Texture and Optical Properties of Donor/Acceptor Complexes. Diindenoperylene/F6TCNNQ vs Alpha-Sexithiophene/F6TCNNQ, J. Phys. Chem. C. 122 (2018) 18705-18714. doi:10.1021/acs.jpcc.8b03744.

[18] W.N. Han, K. Yonezawa, R. Makino, K. Kato, A. Hinderhofer, R. Murdey, R. Shiraishi, H. Yoshida, N. Sato, N. Ueno, S Kera, Quantitatively identical orientation-dependent ionization energy and electron affinity of diindenoperylene, Appl. Phys. Lett. 103 (2013) 253301. doi:10.1063/1.4850531.

[19] H. Fukagawa, H. Yamane, T. Kataoka, S. Kera, M. Nakamura, K. Kudo, N. Ueno, Origin of the highest occupied band position in pentacene films from ultraviolet photoelectron spectroscopy: Hole stabilization versus band dispersion, Phys. Rev. B - Condens. Matter Mater. Phys. 73 (2006) 24-26. doi:10.1103/PhysRevB.73.245310.

[20] N. Sato, H. Inokuchi, I. Shirotani, Polarization energies of tetrathiafulvalene derivatives, Chem. Phys. 60 (1981) 327-333. doi:10.1016/0301-0104(81)80168-1.

[21] A.C. Dürr, F. Schreiber, M. Münch, N. Karl, B. Krause, V. Kruppa, H. Dosch, High structural order in thin films of the organic semiconductor diindenoperylene, Appl. Phys. Lett. 81 (2002) 2276-2278. doi:10.1063/1.1508436.

[22] M.M. Payne, J.H. Delcamp, S.R. Parkin, J.E. Anthony, Robust, Soluble Pentacene Ethers, Org. Lett. 6 (2004) 16091612. doi:10.1021/ol049593z.

[23] T. Okamoto, M.L. Senatore, M.-M. Ling, A.B. Mallik, M.L. Tang, Z. Bao, Synthesis, Characterization, and Field-Effect Transistor Performance of Pentacene Derivatives, Adv. Mater. 19 (2007) 3381-3384. doi:10.1002/adma.200700298.

[24] O.L. Griffith, J.E. Anthony, A.G. Jones, D.L. Lichtenberger, Electronic properties of pentacene versus triisopropylsilylethynyl- substituted pentacene: Environment-dependent effects of the silyl substituent, J. Am. Chem. Soc. 132 (2010) 580-586. doi:10.1021/ja906917r.

[25] Y. Sakamoto, T. Suzuki, M. Kobayashi, Y. Gao, Y. Fukai, Y. Inoue, F. Sato, S. Tokito, Perfluoropentacene: HighPerformance p-n Junctions and Complementary Circuits with Pentacene, J. Am. Chem. Soc. 126 (2004) 8138-8140. doi:10.1021/ja0476258.

[26] H. Meng, M. Bendikov, G. Mitchell, R. Helgeson, F. Wudl, Z. Bao, T. Siegrist, C. Kloc, C.-H. Chen, Tetramethylpentacene: Remarkable Absence of Steric Effect on Field Effect Mobility, Adv. Mater. 15 (2003) 10901093. doi:10.1002/adma.200304935.

[27] R. Schmidt, S. Göttling, D. Leusser, D. Stalke, A.-M. Krause, F. Würthner, Highly soluble acenes as semiconductors for thin film transistors, J. Mater. Chem. 16 (2006) 3708-3714. doi:10.1039/B607172D.

[28] M.L. Tang, T. Okamoto, Z. Bao, High-Performance Organic Semiconductors: Asymmetric Linear Acenes Containing Sulphur, J. Am. Chem. Soc. 128 (2006) 16002-16003. doi:10.1021/ja066824j.

[29] L. Lyons, Energy gaps in organic semiconductors derived from electrochemical data, Aust. J. Chem. 33 (1980) 1717. doi:10.1071/CH9801717.

[30] B. Shen, T. Geiger, R. Einholz, F. Reicherter, S. Schundelmeier, C. Maichle-Mössmer, B. Speiser, H.F. Bettinger, Bridging the Gap between Pentacene and Perfluoropentacene: Synthesis and Characterization of 2,3,9,10Tetrafluoropentacene in the Neutral, Cationic, and Dicationic States, J. Org. Chem. 83 (2018) 3149-3158. doi:10.1021/acs.joc.7b03241.

[31] J. Sworakowski, How accurate are energies of HOMO and LUMO levels in small-molecule organic semiconductors determined from cyclic voltammetry or optical spectroscopy?, Synth. Met. 235 (2018) 125-130. doi:10.1016/j.synthmet.2017.11.013. 